\title{
De la ciencia deductiva de Sherlock Holmes al mundo de la cotidianidad de Alfred Schütz: una reflexión en torno a la relación sujeto-objeto ${ }^{1}$
}

\section{From the deductive science of Sherlock Holmes to Alfred Schütz's world of everyday life: a reflection on the subject- object relationship}

Recibido: 19 de abril de 2015 - Revisado: 30 de septiembre de 2015 - Aceptado: 12 de abril de 2016

Rodrigo Moreno Aponte ${ }^{2}$

\section{Resumen}

El artículo hace una analogía entre las investigaciones del protagonista de las novelas policíacas de Conan Doyle, Sherlock Holmes, y la epistemología. De ahí se pone en evidencia la distancia entre el sujeto y objeto. Se toma una postura crítica frente a esto, para sustentar la idea del vínculo del investigador con su objeto de estudio desde la propuesta fenomenológica del mundo de la cotidianidad de Alfred Schütz. Se expone la distancia que el sociólogo austriaco tiene de las ideas de Husserl, y que le permiten relacionar sus planteamientos con la acción social en la investigación en ciencias sociales.

\section{Palabras clave}

Ciencias sociales, fenomenología, sujeto, objeto, cotidianidad.

\begin{abstract}
The article makes an analogy between the investigations of the protagonist of the detective novels of Conan Doyle, Sherlock Holmes, and epistemology. Hence the distance between the subject and the object becomes evident. We take a critical stance against this, to support the idea of the link of the researcher with his object of study from the phenomenological proposal of the world of the daily life of Alfred Schütz. It explains the distance that the Austrian sociologist has from Husserl's ideas, and that allows him to relate his approaches to social action in social science research.
\end{abstract}

Keywords

Social sciences, phenomenology, subject, object, everyday life.

\footnotetext{
Este artículo forma parte del proyecto de investigación, "Pedagogía hermenéutica del lugar. Narrativas del exterior urbano de la escuela en su vínculo con el desarrollo de la alteridad en la acción educativa", que corresponde a una revisión de la sección metodológica de la tesis para optar como Doctor en Educación y Comunicación Social de la Universidad de Málaga, España.

2 Doctor en Educación y Comunicación Social, Universidad de Málaga, España. Magíster en Filosofía, Pontificia Universidad Javeriana, Bogotá, Colombia. Licenciado en Humanidades y Lengua Castellana, Universidad Distrital "Francisco José de Caldas", Bogotá, Colombia.

Correo electrónico: rodrigomorenoaponte@hotmail.com Para citar este artículo use: Moreno, R. (2016). De la ciencia deductiva de Sherlock Holmes al mundo de la cotidianidad de Alfred Schütz: una reflexión en torno a la relación sujetoobjeto. Civilizar Ciencias Sociales y Humanas, 16(31), 177-190.
} 


\section{Introducción}

En la actualidad las ciencias sociales y las humanidades pasan por una coyuntura global en la que, debido a los intereses de los Estados por mantener un estatus económico que los mantenga a la par de lo que exige el mundo en esta dinámica, se han visto relegadas a un segundo plano, e incluso eliminadas de los intereses de distintas universidades en sus eventos, proyectos, presupuestos y planes de estudio. Tal y como lo muestra Martha Nussbaum (2010), pareciera que la impronta fuera que los campos de conocimiento distintos a los de las ciencias duras no fueran necesarios para el desarrollo social. Ahora, más que nunca, es que los investigadores en las áreas que se acusan de inconmensurabilidad con respecto a la rigurosidad científica, necesitan poner en evidencia sus argumentos frente a la forma como acceden al conocimiento social.

Así pues, este escrito, más que mostrar un desarrollo en el campo de las ciencias sociales, lo que busca es afirmar una postura investigativa que se viene dando en las últimas décadas, en oposición a un sentir positivista que intenta alejar al investigador de su experiencia vital y el mundo de la vida cotidiana. Tampoco se pretende acá evidenciar una posición maniquea entre las ciencias sociales y las ciencias duras. Reiteramos, queremos poner de manifiesto que el modo de acceso al conocimiento desde lo que se expone aquí, es una forma precisa para comprender esos fenómenos que les son vedados a las corrientes positivistas dada su teleología.

Por esto último, consideramos pertinente acudir a las ideas de Alfred Schütz, dada la revalorización que su propuesta fenomenológica está teniendo para el desarrollo investigativo de las ciencias sociales (Cristiano, 2012). Además de esto, la importancia que le damos a Schütz tiene que ver con los consensos y disensos en torno a su obra actualmente, lo cual renueva el interés por sus planteamientos. Como expone López (2014), la obra de Schütz ha sido interpretada a la luz de la oposición entre subjetivismo y objetivismo, no obstante, la idea de que él da preponderancia a la parte subjetiva, no sería exacta.

El asunto fundamental es el de la relación entre el substrato originario de las experiencias humanas y los intentos de tratarlo (come to terms with) cognitiva y teóricamente. En este sentido, Schütz presta atención de igual manera a ambos lados de la cuestión (p. 177).

Así, estableciendo el vínculo entre los dos, se puede superar el objetivismo. También encontramos debates vigentes acerca del proyecto fenomenológico y sociológico de Schütz. En algunos casos se observa que los intérpretes de su obra se refieren a su propuesta investigativa con el nombre de fenomenología sociológica, sociología fenomenológica o fenomenología social. Toledo (2012) apuesta por la expresión "programa sociofenomenológico", para lograr un equilibrio entre los nombres anteriores; no obstante, nos recuerda que Schütz

[...] admite que su programa podría llamarse psicología intencional o psicología fenomenológica e, igualmente, el rótulo de sociología fenomenológica o sociología comprensiva le son adecuados. A su parecer la cuestión de la etiqueta no es mayormente relevante, lo que importa es investigar de manera exhaustiva el fenómeno de la intersubjetividad y de la socialidad (Toledo, 2009, p. 75).

Este tipo de planteamientos que se están revitalizando alrededor de la obra de Schütz, nos sirve como fondo para establecer puntalmente lo que nos proponemos explicar en este artículo, a saber: en la fenomenología aplicada a las ciencias sociales, la función del investigador-observador excede la relación sujeto-objeto de las ciencias duras. Así pues, usaremos un recurso que incomoda a los defensores de la verificabilidad del objeto de estudio, pero que cobra relevancia en una reflexión sobre la fenomenología, esto es, el uso 
de escritos literarios como forma de explicación de un fenómeno (Van Manen, 2003).

A manera de introducción, usaremos la figura de Sherlock Holmes para dar una mirada a la situación epistemológica de la ciencia deductiva. Luego se hará un acercamiento a las ciencias exactas desde la óptica de Husserl. Finalmente, se realizará un vínculo con Schütz, desde su concepto de mundo de la vida cotidiana.

\section{La ciencia de la deducción de Sherlock Holmes}

Para empezar, acudiremos a uno de los protagonistas literarios más importantes en el campo de la investigación criminal: Sherlock Holmes. Este investigador privado es reconocido por hacer uso de su innegable genialidad para resolver los casos más obscuros y complejos. Para lograr el éxito de sus investigaciones lleva a la práctica lo que su autor, Conan Doyle (2007), titula en uno de sus libros como: "La ciencia del razonamiento deductivo". Para Holmes, la investigación "es, o debería ser, una ciencia exacta, y se la debe tratar del mismo modo frío y sin emoción" (p. 15).

Sin pudor alguno, Holmes reconoce su ingenio. Evidencia los vacíos de algunos de sus colegas que se dejan llevar por sus sentidos, y son engañados ingenuamente por los datos de la experiencia sensible. De algunos de ellos destaca que poseen la intuición, pero que desconocen la gama de conocimientos exactos. Para contrarrestar esto, Holmes plantea tres facultades necesarias para un detective ideal: capacidad de observación, deducción y conocimientos exactos.

Holmes, en un diálogo con su fiel amigo Watson, demuestra cómo funcionan estos tres tipos de facultades mentales. Solo con observarlo, le indica que estuvo en una oficina de correos enviando un telegrama. Argumenta que para llegar a este tipo de conclusiones es necesario establecer que la observación y la deducción son dos procesos mentales distintos:

La observación me dice que lleva usted un pegotito rojizo pegado al borde de la suela. Justo delante de la oficina de correos de Wigmore Street han levantado el pavimento y han esparcido algo de tierra, de tal modo que resulta difícil no pisarla al entrar. La tierra tiene ese peculiar tono rojizo que, por lo que yo sé, no se encuentra en ninguna parte del barrio. Hasta aquí llega la observación. Lo demás es deducción.

— ¿Y cómo dedujo lo del telegrama?

-Pues, para empezar, sabía que no había escrito una carta, porque estuve sentado frente a usted toda la mañana. Además, su escritorio está abierto y veo que tiene usted un pliego de sellos y un grueso fajo de tarjetas postales. Así pues, ¿a qué iba a entrar a la oficina de correos si no era para enviar un telegrama? Una vez eliminadas todas las posibilidades, la única que queda tiene que ser la verdadera (p. 15).

A través de este tipo de razonamientos lógicos encontramos cómo el detective inglés resuelve distintos enigmas. En su método se intuye, además, un modelo cartesiano que nos indica que los sentidos obnubilan la observación e inducen al error. Las emociones son enemigas del razonamiento claro. Al detective no le interesa relación alguna con los protagonistas, más allá de lo que la evidencia de los casos le permite deducir y observar. Diríamos que las personas involucradas en sus investigaciones son meros datos que aportan pistas para la resolución del problema.

Si bien el individuo es un rompecabezas insoluble, cuando forma parte de una multitud se convierte en una certeza matemática. Por ejemplo, nunca se puede predecir lo que hará un hombre cualquiera, pero se puede decir con exactitud lo que hará la población por término medio. Los individuos varían, pero los porcentajes se mantienen constantes. Eso dicen los expertos en estadística (p. 121). 
"Deduciríamos" de este fragmento una admiración por el individuo en tanto dato. En uno de los casos más complejos que tuvo que resolver el detective inglés, debido a la falta de pruebas, es decir, la ausencia de objetos que puedan pasar por los procesos mentales ya planteados, decide retirarse a una montaña cercana a los sucesos del crimen. Esto, para estar al tanto de los movimientos de las personas sin ser visto. Se convierte en un observador pasivo a la espera de que su posición arroje los resultados que le ayuden a solucionar el problema.

La analogía que queremos mostrar es que aquí, el observador Holmes, se equipararía a un científico natural, porque es alguien que se aleja y no participa en pautas de interacción.

Los hechos, datos y sucesos que debe abordar el especialista en ciencias naturales son hechos, datos y sucesos solamente dentro del ámbito de observación que le es propio, pero este ámbito no "significa" nada para las moléculas, átomos y electrones que hay en él (Schütz, 1974, p. 37).

Al establecerse este distanciamiento no se probaría un interés por el Otro, a pesar de que Holmes comparte con él el interés por la resolución de los crímenes. El Otro no se revela en sus métodos, en gran medida, porque de seguirlos, no llevaría a cabo la resolución de los crímenes, tal y como lo presenta el método de la ciencia de la deducción.

Es de la máxima importancia -dijo- no permitir que las cualidades personales influyan en nuestra capacidad de juicio. Para mí, un cliente es una mera unidad, un factor del problema. Las cuestiones emocionales son enemigas del razonamiento claro. Le aseguro que la mujer más fascinante que jamás he conocido fue ahorcada por haber envenenado a tres niños para cobrar un seguro, y que el hombre más repelente que conozco es un filántropo que lleva gastado casi un cuarto de millón en ayudar a los pobres en Londres (Doyle, 2007, p. 26).
Holmes puede acceder a su objeto, mas no al sujeto. Puede aprender las características de un criminal a partir de sus deducciones, pero solo las cuestiones sobre cómo llevó a cabo el crimen. ¿Qué acciones lo motivaron? ¿Cuál fue la intención? Son respuestas que escapan a la lógica holmesiana. Para conocerlas, no son pocas las ocasiones que tiene que dirigirse al propio individuo y acercarse en el cara-a-cara para preguntarle por su motivación.

En un pasaje del libro El sabueso de los Baskerville (Doyle, 2007), Holmes deduce la personalidad de un sujeto que no conoce, con tan solo ver un bastón que ha olvidado la persona en mención. Los datos arrojan que el individuo es médico; el bastón fue un regalo que le brindó al momento de irse de un hospital en el que laboró, y de ahí pasó a trabajar en el campo; es cirujano o médico interno, un poco más allá de un estudiante posgraduado; se fue del hospital hace cinco años; su personalidad es afable, poco ambicioso y distraído; dueño de un perro de estatura media con respecto a su género. Cuando conoce al individuo y lo confronta cara a cara, Holmes reconoce que no es físicamente como lo imaginaba y, más importante, el bastón fue regalado por un amigo del hospital en conmemoración de su matrimonio. Un error en la observación y deducción.

Más diciente es el momento en el que tiene la certeza de quiénes son y dónde se ubican los responsables de un crimen. Al momento de ir a aprehenderlos le dirige las siguientes palabras al inspector de la policía: "me gustaría que el propio Jonathan Small -el criminal que está a punto de atrapar- me explicara algunos detalles del caso. Ya sabe usted que me gustaría dejar resueltos mis casos hasta el último detalle" (Doyle, 2007, p. 113). Es decir, Holmes, aunque ha resuelto el caso, quiere saber las motivaciones del criminal. Estas respuestas escapan a cualquier observación o deducción que haya podido hacer durante la resolución del caso. El método de Holmes busca ir a las cosas mismas, pero existe una barrera que no deja que lo consiga. 
La deducción sirve para sacar datos del individuo sin que necesariamente haya una interacción con él. Es obvio que para los fines de esclarecer un crimen basta con las geniales deducciones de Holmes. No obstante, las motivaciones e intenciones del sujeto dejarían de estar al alcance del observador que se aleja en su montaña de conocimientos, observaciones y deducciones lógicas.

Así como lo hace Sherlock Holmes, la ciencia natural desconoce el acceso a las cosas mismas debido a su búsqueda de objetividad y dominio universal de los fenómenos. A la par de esta afirmación se pone de relieve la oposición del método fenomenológico, del que Husserl afirma:

La fenomenología procede aclarando visualmente, determinando y distinguiendo el sentido. Compara, distingue, enlaza, pone en relación, hace trozos o separa partes no independientes; pero todo puramente viendo. No construye teorías ni matematiza; no lleva a cabo, en efecto, explicaciones en el sentido de la teoría deductiva (Husserl, 1982, p. 72).

Husserl indica cómo la ciencia natural falla en su pretensión de dominio sistemático de los fenómenos. La ciencia separa lo objetivo de lo subjetivo de un fenómeno. La experiencia de los sujetos, antes de la relación con el objeto de estudio, no tendría valor si consideramos su meta. Así se hace evidente la preocupación del filósofo alemán:

Es ahora de la mayor importancia considerar el desplazamiento, consumado ya por Galileo, en virtud de que el mundo matemáticamente cimentado de las idealidades pasó a convertirse en el único mundo real, el mundo efectivamente dado como perceptible, el mundo de la experiencia real y posible; en una palabra: nuestro mundo de vida cotidiano. Este desplazamiento sustitutorio fue heredado bien pronto por sus sucesores, los físicos de todos los siglos subsiguientes (Husserl, 1991, p. 50).

No obstante, la relación entre el campo de objetividad y la constitución de lo sensible genera una falacia. Las demostraciones de la ciencia se deben dar en el campo de la experiencia, el campo donde se da esa subjetividad que busca dejar de lado. En este sentido, Javier San Martín (1986) explica:

El hecho de que lo verificable tenga que ser isomorfo con la única experiencia posible para el hombre, significa que las teorías de la ciencia natural solo tienen valor cuando son convertibles en hechos concretos de la experiencia, para lo cual, en algún momento de su desarrollo han de volver a ser traducidas a los términos de la experiencia ordinaria (p. 65).

Nuestra posición con respecto a la vía holmesiana, la de la ciencia natural, no es en lo absoluto descalificadora, solamente busca evidenciar un proceso entendido desde la ciencia positiva en la relación sujeto-objeto, que es distinto al que recorre una perspectiva fenomenológica. Dicho esto, podemos establecer que una noción positivista puede ser necesaria para su objeto de estudio, pero, que en el caso de la analogía entre Holmes y la ciencia natural, se sitúa en la imposibilidad de comprender las intenciones de los hechos en los cuales se da la interacción social.

Aun cuando los enigmas son, hablando en general, inmanentes en principio a la ciencia de la naturaleza, es obvio que sus soluciones de acuerdo a las premisas y las conclusiones son, en principio, trascendentes a las mismas. Se caería en un círculo vicioso si se esperase de la ciencia de la naturaleza misma la solución de todo problema que se refiere a ella como tal (Husserl, 1962, p. 21).

Sin duda el método de las ciencias naturales es plausible para descubrir datos, confrontarlos, llegar a conclusiones y hallar el camino de la veracidad. Aunque, mientras que las ciencias son incapaces de la fundamentación de un conocimiento de la totalidad de la experiencia humana, la fenomenología busca llegar en verdad al ideal que está vedado a la pretensión positivista de la cientificidad. 
Encontramos este mundo como mundo de todas las realidades conocidas y desconocidas. A él, al mundo de la intuición realmente experimentante pertenece la forma espaciotemporal con todas estas formas corporales que deben ser dispuestas ordenadamente en él; en él vivimos según nuestros modos de ser personales- corporales. Pero aquí no encontramos nada de las idealidades geométricas, no encontramos el espacio geométrico ni el tiempo matemático en todas sus formas (Husserl, 1991, p. 52).

La búsqueda de ese conocimiento vedado para las ciencias naturales, pero accesible a la fenomenología, se suele suscribir con la máxima: "a las cosas mismas". Esas cosas mismas serían las que no contempla la ciencia.

Cualquier posible intento por parte de un círculo de investigadores no específicamente matemático ni científico-natural, de llevar al científico a este tipo de reflexiones, es rechazado como "“metafísica"! El especialista que ha consagrado su vida a estas ciencias es, en fin $-\mathrm{y}$ esto tiene para él el carácter de lo obvio-, quien mejor puede saber lo que en y con su trabajo se propone y consigue. Las necesidades filosóficas despertadas también en estos investigadores por motivos históricos que deberán ser aún esclarecidos (necesidades "matemático-filosóficas", "científico-naturalfilosóficas"), son suficientemente satisfechas por ellos mismos, pero, a decir verdad, de un modo tal que la dimensión entera en la que hay que penetrar con ánimo indagatorio no es siquiera percibida, ni menos, por tanto, cuestionada (Husserl, 1991, p. 59).

Dijimos que asumíamos de manera crítica la forma como, en cierta ocasión, Holmes se distanciaba del mundo para resolver un crimen. Destacábamos la distancia que él tenía hacia el mundo, debido al carácter de dato que le daba al sujeto. Sin embargo, no nos incomoda del todo esta forma de alejamiento del observador, ya que, de hecho, es necesaria para la cuestión fenomenológica que planteamos acá; obviamente con unos matices particulares.
En la fenomenología en ciencias sociales, esta observación, aunque se separa del objeto, no está señalando que exista un mundo aparte, o que deba alejarme de él para captar al objeto. Si fuera así estaríamos cayendo en el objetivismo. Más bien, lo que se quiere establecer, es que el alejamiento nos lleva al sentido opuesto, al de la subjetividad. Schütz define este alejamiento como el "desinterés" o distanciamiento del observador, de la siguiente manera:

Este no comparte las esperanzas y temores del actor acerca de si se comprenderán el uno al otro y lograrán su objetivo mediante el entrelazamiento del motivo. Así, su sistema de significatividades difiere del de las partes interesadas y le permite al mismo tiempo ver más y menos de lo que estas ven. Pero en todas las circunstancias, lo accesible a su observación son solamente los fragmentos manifiestos de las acciones de ambos asociados. Para comprenderlos, el observador tiene que valerse de su conocimiento de pautas típicamente similares, y tiene que construir los motivos de los actores a partir de ese fragmento del curso de acción que está abierto a su observación (Schütz, 1974, p. 54).

Es aquí donde sigue desbaratándose la dicotomía sujeto-objeto. Aquí se da un proceso en el que dejo que el "objeto" me muestre sus datos, pero así mismo, doy un paso atrás del mundo. Vuelvo a un estado de reflexión en el cual cuestiono mis propios prejuicios. Esto no ocurre en la teleología de las ciencias duras, según Schütz. Al respecto, haciendo alusión al fenomenólogo vienés, afirma López (2008):

Los especialistas en ciencias de la cultura, cegados por el naturalismo, han pretendido buscar las verdades "objetivas" sin indagar las actividades subjetivas de la mente, únicas a partir de las cuales se constituye el sentido óntico del mundo de la vida que es dado de antemano (p. 83).

Osorio (1999) de manera muy clara, utiliza el ejemplo del asentamiento de una hidroeléctrica en unos terrenos indígenas, y la 
idea que de esta situación tienen un antropólogo social, ecologistas, dueños de la hidroeléctrica e indígenas. Esta descripción nos ayuda para sostener nuestra idea:

El científico al reflexionar se da cuenta que puede poner en tela de juicio los argumentos de ecologistas e indígenas, luego los suyos como antropólogo social. Incluso puede dudar de la existencia de la central hidroeléctrica, es decir, puede poner entre paréntesis al mundo, suspender sus juicios respecto de él. Sin embargo, se da cuenta que es él quien está reflexionando, que toda experiencia acerca del mundo pasa por sí mismo como sujeto y que hay algo en él que da sentido al mundo (p. 123).

Desde nuestra perspectiva nos distanciamos de la idea del alejamiento total del investigador. Es decir, consideramos que es necesario volver y tener un contacto con el mundo, para, de esta manera, poder determinar la relación con el objeto de estudio. Si no fuera así, cosificaríamos la experiencia que queremos interpretar y la reduciríamos a un mero dato procesado por medio de nuestra metaexperiencia. ¿Queremos decir con esto que debemos tener una relación directa con el mundo sensible?

Para responder esto último, hagamos un alto y volvamos a Holmes. Dentro de la construcción literaria de Conan Doyle encontramos lo que se podría llamar la parte sensible de las aventuras del detective inglés: Watson, su gran compañero. Sensible, no en un sentido romántico, sino en la vía de considerar otras posibilidades que, al ser confrontadas con las deducciones lógicas e irrefutables, pierden su valor para el Holmes epistemólogo. En este sentido podríamos decir que Holmes busca la objetividad como una característica inherente a sus teorías. Mientras tanto Watson está en un campo poco provechoso para la ciencia de la deducción y su observación subsecuente: está en la cuestión de la subjetividad, siempre en desventaja con la situación objetiva.

En contraste, una consideración "subjetiva" es aquella que ha sido dejada de lado, o lo sería, o debería serlo, por las personas que han participado en la discusión de forma racional -la que se considera, o debería considerarse, que es irrelevante para el objeto material de la teoría-. Decir que alguien está introduciendo consideraciones "subjetivas" en una discusión donde se exige objetividad es, en términos generales, decir que está introduciendo consideraciones que otros estiman que están fuera de lugar (Rorty, 1989, p. 232).

Es de destacar que Holmes sí tiene en cuenta las cuestiones subjetivas, ya que estas forman parte de la objetividad que busca. ¿Acaso podríamos establecer que algo es objetivo, sin establecer qué es subjetivo? Siguiendo a Rorty (1989),

[...] juzgar la subjetividad es tan arriesgado como juzgar la relevancia. En un sentido más tradicional, "subjetivo" contrasta con "que corresponde a lo que está ahí fuera", y, por tanto, significa algo parecido a "producto únicamente de lo que hay aquí dentro" (p. 232).

Holmes y Watson son amigos, pero van por caminos distintos buscando un mismo fin: la verdad. La objetividad no se opone a la subjetividad, y en el plano que nos ocupa, buscarían un estadio de conocimiento similar. Fuera del ámbito de las demostraciones lógicas en el mundo de Holmes, no se podrían resolver estos misterios. Y está bien, porque estamos en un campo de investigación criminal. Pero seguramente no sirve para explicar el sentido de un poema, de una novela o de la experiencia vivida. Aquí nos jugamos la idea que queremos sustentar en este escrito. Si no nos vamos por el lado del Holmes epistemólogo, entonces ¿estaremos de parte del Watson de las sensaciones e intuiciones?

En el contexto de los personajes literarios que están dentro de investigaciones policiales, destacan otros que no utilizan los medios geniales de Holmes. Ellos tienen que recurrir a otros procesos mundanos para acceder a la solución de los crímenes. De los múltiples 
personajes que podemos encontrar en esta vía, haremos referencia brevemente a uno en particular.

En la novela Plenilunio de Antonio Muñoz Molina (1997), se presenta al personaje principal como un detective que tiene que investigar la cruel muerte de una niña. Sus investigaciones no son fructíferas y las pistas no dan mayores luces acerca del crimen. Una segunda víctima sobrevive, una niña de once años. Convive con ella de forma tal que la conoce en su vida cotidiana, como vemos a continuación:

Se ponía de puntillas para darle un beso y salía sola del portal, como le habían enseñado que lo hiciera, echaba a andar delante de él, camino del colegio, con la mochila a la espalda, con un chubasquero amarillo y un paraguas rosa los días de lluvia, con botas amarillas de goma. De vez en cuando volvía un instante la cabeza hacia el inspector, nada más para estar segura de que la seguía y la cuidaba, pero que si se encontraba con otras niñas obedecía las instrucciones recibidas y actuaba con una desenvoltura perfecta, sin mirar hacia atrás, o haciéndolo de un modo tan hábil que nadie sospecharía con el vínculo del hombre alto y canoso que caminaba a una cierta distancia, fijo siempre en ella, sin perderla de vista hasta que desaparecía en el interior del colegio, en el tumulto de niñas y niños y madres de todas las mañanas (p. 386).

Esta es una ruta de investigación totalmente opuesta a la de Holmes. Es un investigador que busca las pistas y tiene que ir directamente a los problemas, a tal punto de convivir con ellos. No se retira a una montaña como el detective inglés, sino que vivencia la experiencia de sus otros. ¿Si esta es una vía contraria a la de Holmes, entonces podríamos decir que este es el tipo de investigación afín a lo que proponemos en este escrito?

El investigador español cede a sus impulsos, no toma distancia de ellos, no son meros datos. Logra dar con el criminal cotejando dis- tintas vivencias que lo llevan a sentir de manera profunda la problemática. La cuestión radica en que este investigador se deja permear por sus sentidos en un extremo tal, que nubla su buen juicio y lo lleva a cometer errores en su investigación. Así como Holmes no puede conocer la esencia de los fenómenos, el detective de Plenilunio no puede saber de estos porque cede a sus impulsos e interpretaciones personales. En síntesis, tanto el uno como el otro no acceden al sentido de los fenómenos que investigan.

En el campo de la investigación social se necesita de un investigador que esté presente en el mundo de la experiencia, para el acceso a las esencias, de una forma objetiva; pero a su vez, que no pierda su presencia. Esto es, un vínculo entre el sujeto-objeto desde el que prima la cuestión del objeto como mero dato de análisis, se diluya.

\section{Investigar en el mundo de la cotidianidad}

Para Schütz la relación entre la actitud natural y la reducción fenomenológica sigue siendo condición necesaria para la investigación en ciencias sociales. En la vida diaria, al tomar como tomo la posición de la actitud (o punto de vista) natural, vivo dentro de los actos mismos de asignación de significado y solo soy consciente de la objetividad constituida en ellos, es decir, el significado objetivo. Solo después que yo, "mediante un esfuerzo penoso", como dice Bergson, me aparto del mundo de los objetos (gegenstände) y dirijo mi mirada a mi corriente interna de conciencia, solo después que "pongo entre paréntesis" el mundo natural y no atiendo más que a mis experiencias conscientes dentro de la reducción fenomenológica, me vuelvo consciente de este proceso de constitución (Schütz, 1972, p. 66).

En la actitud natural bastaría con la referencia al significado objetivo de la persona, así ella pueda presentar significaciones subjetivas del objeto en un plano meramente 
superficial. Acá se hace necesaria la presencia de la reducción fenomenológica que nos lleva al estado de epojé que, en el contexto del sociólogo vienés, no nos pone entre paréntesis del mundo entero, sino que nos remite a la subjetividad del pensador. Al respecto nos dice Leal (2007):

Recordemos que el observador debe tener presente que es portador de una carga cultural que forma parte de sí, a propósito de los diversos procesos de socialización de los cuales ha sido objeto $\mathrm{y}$, si tiene la pretensión de conocer al "Otro", no queda otra alternativa sino dejar de lado las categorías interpretativas que utiliza en el mundo cotidiano (p. 220).

El mundo de la vida sigue subsistiendo como forma de contemplación teórica. Esta epojé la sintetiza Schütz (1974) así:

1) La subjetividad del pensador como un hombre entre sus semejantes, incluyendo su existencia corporal como ser humano psicofísico dentro del mundo; 2) el sistema de orientación por el cual el mundo de la vida cotidiana es agrupado en zonas que están dentro del alcance actual, recuperable, posible, etc.; 3) la ansiedad fundamental y el sistema de significatividades pragmáticas que en ella se originan (p. 230).

De todas formas, es importante enunciar que Schütz tenía claro que el proyecto husserliano le servía hasta cierto punto, como lo evidencia claramente a continuación.

El propósito de esta obra, que consiste en analizar el fenómeno del significado de la vida social ordinaria (mundanen), no requiere que se alcance un conocimiento trascendental que vaya más allá de esa esfera, o que sigamos manteniéndonos dentro de la zona de la reducción fenomenológica trascendental. Con una condición, sin embargo: que no es mantenernos como "psicólogos fenomenológicos" (Schütz, 1972, p. 73).

El distanciamiento se constata en un alejamiento de la trascendentalidad, es decir que, el proyecto filosófico al cual le apuntaba Husserl, pierde interés para Schütz. No queremos decir con esto que el problema filosófico no esté presente, ya que este permea todas las ideas del investigador austriaco; sin embargo, está claro que su fin es el estudio de la vida en la experiencia mundana.

Schütz no está negando la importancia del análisis trascendental husserliano, tan solo dice que para estudiar el ámbito de la vida social, enmarcada en el ámbito de intencionalidad que Husserl llamó actitud natural, no es necesario incursionar en la dimensión trascendental que sigue teniendo vigencia en la esfera de la fenomenología eidética profunda (Toledo, 2009, p. 77).

Es en el contexto de las ciencias sociales donde se focaliza su atención para explicar las formas de construcción de significado en las relaciones sociales. Para esto, en la misma vía de la crítica que hace Husserl a la matematización y sus consecuencias en las ciencias naturales, Schütz enfila sus argumentos para defender su posición en contra del positivismo. Trataremos de sintetizar esto último en los siguientes puntos:

1. Entendiendo por realidad social el cúmulo de hechos del mundo social cultural dentro del cual se dan relaciones complejas, las ciencias sociales son un conocimiento organizado de esta.

2. Sería un error tratar de acceder al conocimiento de la realidad social a través de categorías presupuestas como el naturalismo y el empirismo lógico. Si se hiciera de esta manera, los problemas conceptuales ya estarían resueltos al momento de abordar la investigación social.

3. Una interpretación de un observador con categorías establecidas indaga por la experiencia de lo que acontece entre los actores, pero deja de lado otras dimensiones de la realidad social, como lo es la experiencia misma de ellos. 
4. El observador, al concentrarse solamente en el acto de percepción sensorial, olvida otras esferas de la realidad. Realiza una fragmentación de la experiencia de forma arbitraria.

Ahora bien, en el plano de orientación del pensador austriaco con respecto al trato de los conceptos que encontramos en Husserl, se reporta su interés por el mundo de la vida y vida cotidiana. Desde una lectura rápida, se podría considerar que estos dos conceptos son afines, sinónimos, o que guardan un mismo sentido, como lo declara Salas (2006) en relación con Schütz y Husserl: "Se mantienen elementos coincidentes, para ambos autores el Mundo de la Vida (cotidiana) se caracteriza principalmente por ser un mundo previo, por su pre-reflexibilidad y por ser el escenario histórico donde se desarrolla la cuestión de la intersubjetividad" (p. 194).

Aunque se puede decir que Schütz introduce el concepto de mundo de la vida a la reflexión epistemológica en ciencias sociales (López, 2013), sostendremos que este no puede ser sinónimo del mundo de la vida cotidiana, ya que, si lo que busca Schütz es afirmar la intersubjetividad en el mundo social, que es aporética en el mundo de la vida, entonces debe existir un desplazamiento. Lo que hace Schütz es afirmar el sentido del mundo de la vida husserliano y tomar una parte de este, a la que se refiere como mundo cotidiano; para ser más exactos: "el mundo de la vida cotidiana".

Schütz considera el mundo de la vida en un sentido amplio, mientras que la vida cotidiana es una parte de la realidad: "El mundo de la vida cotidiana es la región de la realidad en la que el hombre puede intervenir y que puede modificar mientras opera en ella mediante un organismo animado" (Schütz \& Luckmann, 1977, p. 25). Es en esa región donde se da la intersubjetividad. Sin embargo, se mantiene la problemática de la actitud natural, en la cual

[...] la posibilidad de una reducción de la experiencia a elementos como estos, y la con- secuente cuestión de cómo llegan a reconstituirme en objetos de experiencia, no se me presenta en la actitud natural. Más bien expresa un problema que pertenece al pensamiento específicamente filosófico y científico (p. 26).

Para Schütz (1972) es en el mundo de la vida cotidiana donde verdaderamente se viene a establecer que "el significado se constituye, por lo tanto, en un fenómeno intersubjetivo" (p. 62). Podríamos aseverar entonces que el acceso al mundo de la vida, truncado por sus ansias de trascendentalidad, abre paso para que Schütz se enfoque en el mundo de la vida cotidiana en el que se construyen las estructuras del mundo social.

La cotidianidad es la realidad preeminente, no es la totalidad del mundo de la vida, sino lo que Schütz denomina: "el mundo total de la vida cotidiana". Ahí, en donde

[...] lo que nos es dado lisa y llanamente en la actitud natural, en ningún caso incluye solamente los objetos de percepción externa (entendidos puramente como tales), sino también los estratos de sentido de orden inferior, gracias a los cuales las cosas naturales son experimentadas como objetos culturales (p. 27).

En este contexto, los objetos de percepción externa son los cimientos del mundo de la vida. De tal manera, el mundo de la vida es la base estructural de la experiencia social que se da en la vida cotidiana.

El mundo de vida es el conjunto de estructuras predicativas que posibilitan el despliegue de la vida social. Entonces, es en la vida cotidiana, donde a través de la acción social y los entramados interactivos que constituye, tiene lugar ese despliegue y donde, a su vez, se genera la (re)producción del mundo de la vida (Estrada, 2000).

\section{Conclusiones}

Schütz se retira de la situación fenomenológica trascendental de Husserl y da paso a 
darle una importancia cardinal a la cotidianidad, donde se desenvuelven sus conceptos de acto y acción, a la vez que le da una pre-eminencia vital a la historicidad del sujeto. La protensión ${ }^{1}$ de la acción, para llegar al acto, necesita de unos medios para alcanzarla; medios que dotan de significado al acto. Una vez más, llegar en la posición de un observador externo que decide las categorías de análisis, implica que se parte de una arbitrariedad para escoger el medio sobre el cual se interpretará la acción, fragmentándola, desconociendo los medios anteriores o futuros, con el propósito de dar una unidad a la acción. En este sentido se pregunta Schütz (1972):

¿De qué sirve hablar acerca del significado a que apunta una acción si ignoramos la fase de la acción que es importante para el actor y la sustituimos, a modo de interpretación, por un segmento arbitrariamente elegido del curso observado que constituye los hechos? (p. 92).

Si considero al Otro en su carácter temporal, quiere decir que no lo tomo como objeto, sino como un cúmulo de vivencias que tienen que desentrañarse.

En este sentido, no consideramos pertinente poder estudiar al sujeto dentro de situaciones controladas para determinar observaciones de su modo de actuar en la sociedad. Un individuo no es el mismo cuando está en una situación controlada a como está en su entorno de acción cotidiana, por lo cual, no se puede deducir su acto en ese sentido. Ahora bien, observemos las siguientes situaciones:

1. En la película El Experimento (2001) se muestra a un grupo de científicos que recluta a voluntarios para confinarlos en un espacio cerrado que está vigilado por cámaras. Las personas que se prestan para esto, cumplen con los roles de policías y presos. La misión de los policías es preservar el orden sin violencia. El experimento se sale de control cuando los carceleros recurren a las agresiones físicas para dominar la situación. Además, frente a los problemas de autoestima de los carceleros y otras anomalías en su comportamiento, se toman su rol de poder de manera extrema.

2. El filme La Ola (2008) muestra a un profesor de secundaria que orienta a los estudiantes para que conformen un grupo particular con todos los integrantes de la clase. Se diferencian del resto mediante el uso de uniformes, un saludo y reglas. En el momento que ellos empiezan a adquirir poder como grupo, se suscitan acciones violentas que obligan al docente a convocar una reunión para dar por terminada la experiencia. Les demuestra que lo que él hizo fue que siguieran una idea por medio de órdenes, sin cuestionarse. El profesor les explica que esta es la base sobre la que se desarrollan las dictaduras. Al final uno de los estudiantes, con poca autoestima, se trastorna por la decisión del maestro. Dispara a sus compañeros porque siente que su mundo se acaba al dar por terminado el grupo en el cual fue, por primera vez, reconocido como un miembro.

El punto en común entre estas dos experiencias es un sujeto que domina su objeto, pero que después se sale de control. En el caso 1 se buscaba escenificar una situación real que, por su misma naturaleza, deja de ser real. Se sale de las manos porque están sometidos a una situación extrema de la que no son capaces de tomar control. Ni investigador ni objeto de investigación se pueden manipular aquí. El caso 2 tiene la misma esencia. El profesor no logra gobernar su experimento particular porque un individuo se salió del control por su experiencia de vida particular.

Si estableciéramos a los investigadores del caso 1 y el profesor del caso 2 como investigadores sociales, se podría dar la razón a Schütz en lo que plantea a continuación:

La relación entre el especialista en ciencias sociales y el títere que ha creado refleja, en cierta medida, un viejo problema de la teología y la metafísica: el de la relación entre Dios y 
sus criaturas. El títere existe y actúa únicamente por gracia del especialista; no puede actuar sino de acuerdo con el propósito que le determina la sabiduría de aquel. Se supone, sin embargo, que actúa no como si estuviera determinado, sino como si pudiera determinarse a sí mismo. Ha sido preestablecida una armonía total entre la conciencia determinada que se asigna al títere y el medio preconstituido dentro del cual se supone que actúa libremente y lleva a cabo elecciones de decisiones racionales. Esta armonía solo es posible porque ambos, el títere y su medio reducido, son creación del hombre de ciencia, que ateniéndose a los principios que lo han guiado, logra en verdad descubrir dentro del universo así creado la perfecta armonía que él mismo ha establecido (Schütz, 1974, p. 70).

¿Qué es un títere? Una cosa a la cual le damos cualidades humanas a partir de los hilos que mueve una persona en particular. No tiene que dialogar o problematizar porque todo está dado según su propia constitución espaciotemporal. Todo está planeado con anterioridad. Pero la persona que mueve los hilos es alguien ausente, su voz no está presente como tal, y si lo está, es a través de una parodia en la cual se enmascara por medio de la actuación del humano-cosa, o de la cosa con cualidades humanas. En tal sentido ambos están ausentes, ni siquiera hay una relación cara-a-cara. No se conocen los unos a los otros. Por lo tanto, consideramos que en las ciencias sociales mover los hilos de otra persona para nuestros fines es cosificar, fragmentar y, de por sí, aniquilar la experiencia del Otro. Una investigación en ciencias sociales que tiene una relación fenomenológica con lo que busca comprender, se resiste a esto.

\section{Notas}

${ }^{1}$ Expresión empleada en este contexto fenomenológico, contrario a retención. "Según se proyecte la extensión intencional del sujeto hacia el pasado o hacia el futuro hablamos, respectivamente de 'retenciones' y 'protensio- nes' como las intencionalidades específicas por medio de las cuales la conciencia es temporal" (Toboso, s.f.).

\section{Referencias}

Becker, C., Maag, N., \& Schneider, A. (Productores), \& Ganssel, D. (Director). (2008). La ola [Película]. Alemania: Rat Pack Filmproduktion.

Conrad. M. (Productor), \& Hirschbiegel, O (Director). (2001). El experimento [Película]. Alemania. Typhon Film / Fanes Film.

Cristiano, J. (2012). Los escritos de Alfred Schütz desde la perspectiva de una teoría de la creatividad de la acción. Revista Universitas Humanística, 75, 305-327. Recuperado de http://www.scielo.org.co/ scielo.php?script $=$ sci_arttext\&pid=S012048072013000100013\&lang=es

Doyle, A. (2007). El signo de los cuatro; El sabueso de los Baskerville. Madrid: Alianza Editorial.

Estrada, M. (2000). La vida y el mundo: distinción conceptual entre mundo de vida y vida cotidiana. Sociológica, 15(43), 103151. Recuperado de http://www.redalyc. org/articulo.oa? $\mathrm{id}=305026539004$

Husserl, E. (1962). La filosofia como ciencia estricta. Buenos Aires: Nova.

Husserl, E. (1982). La idea de fenomenología. México: fondo de cultura económica.

Husserl, E. (1991). La crisis de las ciencias europeas y la fenomenología trascendental. Una introducción a la fenomenología filosófica. Barcelona: Editorial Crítica.

Leal, R. (2007). Aportes teórico-metodológicos de la fenomenología al desarrollo cuali- 
tativo de las ciencias sociales en Alfred Schütz. Alpha (Osorno), 25, 215-225. doi: 10.4067/S0718-22012007000200015

López, D. (2013). Filosofía y Ciencias Sociales. El diálogo intelectual entre Alfred Schutz y Talcott Parsons. Enfoques, 25(2), 6992. Recuperado de http://www.scielo. org.ar/scielo.php?script=sci_arttext\&pi$\mathrm{d}=\mathrm{S} 1669-27212013000200005 \&$ lang $=$ es

López, D. (2014). El problema del objetivismo en la producción de conocimiento sociológico: La correspondencia entre Alfred Schutz, Talcott Parsons y Harold Garfinkel. Cinta Moebio, 51, 171-191. doi: 10.4067/S0717-554X2014000300005

López, G. (2008). El problema de la subjetividad en la explicación sociológica. Una mirada a partir del debate Schutz-Parsons. Revista de estudios sociales, 31, 72-83. Recuperado de http://www.scielo. org.co/scielo.php?script=sci_arttext\&pi$\mathrm{d}=$ S0123-885X2008000300006\&lang=es

Muñoz, A. (1997). Plenilunio. Madrid: Alfaguara.

Nussbaum, M. (2010). Sin fines de lucro ¿Por qué la democracia necesita de las humanidades?. Buenos Aires: Katz editores.

Osorio, F. (1999). El Científico Social entre la Actitud Natural y la Actitud Fenomenológica. Cinta de Moebio, 5, 119-128. Recuperado de http://dialnet.unirioja.es/ servlet/articulo? codigo $=304224 \&$ orden $=$ 66812\&info $=$ link

Rorty, R. (1989). La filosofía y el espejo de la naturaleza. Madrid Cátedra.
Salas, R. (2006). El mundo de la vida y la fenomenología sociológica de Schütz. Apuntes para una filosofía, de la experiencia. Revista de Filosofia, 15, $167-$ 199. Recuperado de http://dialnet.unirioja. es/servlet/articulo?codigo $=2293997$

San Martín, J. (1986). La estructura del método fenomenológico. Madrid: Uned.

Schütz, A., (1972). La construcción significativa del mundo social: introducción a la sociología comprensiva. Barcelona: Paidós.

Schütz, A. (1974). El problema de la realidad social. Buenos Aires: Amorrortu Editores.

Schütz, A., \& Luckmann, T. (1977). Las estructuras del mundo de la vida. Buenos Aires: Amorrortu Editores.

Toboso, M. (s.f.). Tiempo y sujeto (II): Sobre una noción de $<<$ Temporalidad $>>$ del sujeto. A parte rei Revista de Filosofía. Recuperado de http://serbal.pntic.mec. es/ cmunoz11/toboso28.pdf

Toledo, U. (2009). El Programa SocioFenomenológico de Investigación. Cinta de Moebio, 35, 67-87 doi: 10.4067/ S0717-554X2009000200001

Toledo, U. (2012). Socio-fenomenología. El significado de la vida social cotidiana. Concepción, Chile: Editorial Pencopolitana.

Van Manen, M. (2003). Investigación educativa $y$ experiencia vivida. Ciencia Humana para una pedagogía de la acción y la sensibilidad. Barcelona: Idea Books S.A. 
